\title{
In Situ Heating of Lunar Soil in the Transmission Electron Microscope: Simulating Micrometeorite Impacts.
}

\author{
Michelle S. Thompson ${ }^{1}$, Thomas J. Zega ${ }^{1}$ and Jane Y. Howe $\mathrm{e}^{2,3}$ \\ ${ }^{1 .}$ Lunar and Planetary Laboratory, Department of Planetary Sciences, University of Arizona, Tucson, AZ. \\ 2. Hitachi High-Technologies Canada Inc. 89 Galaxy Blvd. Suite 14, Rexdale, ON, Canada. \\ 3. Department of Materials Science and Engineering, University of Toronto, 27 King's College Circle, \\ Toronto, ON, Canada.
}

Mineral grains on the surfaces of airless bodies are continually modified due to their exposure to interplanetary space. They experience radiation processing from solar energetic ions and collisional processes such as micrometeorite impacts. These processes are collectively known as space weathering and together they alter the microstructural, chemical, and optical characteristics of minerals on planetary surfaces [1]. These features were first identified in lunar soils returned by the Apollo missions, e.g., [2]. Understanding the modification of crystal structure and chemistry as a result of space weathering provides insight into the evolution of planetary surfaces throughout the history of the solar system, and is important for determining the mineralogy of those surfaces from spectroscopic remote sensing spacecraft data.

Space weathering features in returned samples from the Moon and asteroids include: nanophase Fe particles (npFe), vesiculated textures, partially and completely amorphous rims, and chemically distinct deposits on grains surfaces, e.g., [2]. These characteristics manifest on the micro- and nano-scales, making the transmission electron microscope (TEM) an ideal tool for their analysis. Since these features were first recognized in returned samples, many groups have attempted to simulate space-weathering processes in the laboratory. Ion irradiation experiments were performed to mimic solar wind bombardment, and light-gas gun impacts, as well as pulse-laser irradiation experiments have been performed to simulate micrometeorite impacts e.g., [3,4]. While these experiments have had varied degrees of success in reproducing the chemical, microstructural, and optical changes that result from natural space weathering, they are all static. Data (images, structural, and chemical information) were collected only before and after the simulated weathering process, not throughout. Here we present a new methodology for simulating micrometeorite impact events, through dynamic in situ heating experiments inside the TEM. This work enables the first direct observation of chemical and structural changes resulting from micrometeorite impact events in real-time.

Grains of mature lunar soil 79221 were suspended in methanol and then drop-cast onto Hitachi SiN thermal e-chips for analysis in the TEM. We thermally shocked these samples to $\sim 1000{ }^{\circ} \mathrm{C}$ and back to room temperature in <1 second inside the Hitachi HF3300 TEM at the University of Toronto. The HF3300 is equipped with bright-field (BF), dark-field (DF), and secondary electron (SE) detectors, a silicon-drift energy-dispersive x-ray spectroscopy (EDS) detector, and a Gatan GIF Quantum electron energy-loss spectrometer (EELS). We recorded video of the heating experiments, and collected BF, high-angle annular dark-field (HAADF), and SE images, as well as EDS maps of the grains before and after heating. We also acquired EELS spectra of several Fe nanoparticles that formed during heating. We acquired spectra with dwell times between 1.0 to $2.0 \mathrm{~s} / \mathrm{px}$ and with a dispersion of $0.25 \mathrm{eV} / \mathrm{channel}$ to determine the oxidation state of $\mathrm{Fe}$ in the nanoparticles. Some samples were thermally shocked multiple times, experiencing as many as 12 simulated impact events. We recorded video throughout 
each heating interval, and imaged and collected EDS maps of the grains after the final heating episode.

During the single thermal shock events, we observed the formation of high densities of nanoparticles both on grain surfaces and in the grain interiors (Fig. 1). The nanoparticles exhibit both euhedral and spherical shapes and EDS mapping revealed they are composed of Fe, suggesting they are analogous to $\mathrm{npFe}$ particles in naturally space-weathered soils. The nanoparticles range in size from <5nm to $\sim 50 \mathrm{~nm}$, with the highest frequency of particles $<15 \mathrm{~nm}$ in size. In grains subjected to multiple heating events, the average size of the npFe particles increased. We also identified elliptical features in the glass matrix as a result of heating that have high contrast in BF images, and low contrast in HAADF images. These features are correlated to surface textural changes in the SE images, consistent with vesicles. EELS spectra were collected for $\mathrm{npFe}$ protruding from the surface of the host grain, enabling measurement of their oxidation state independent from thickness effects or contributions from Fe in the matrix (Fig. 2). EELS measurements indicate that at least a portion of the nanoparticles are composed of $\mathrm{Fe}^{0}$.

These experiments produce features, including $n p F e$ and vesicles, in lunar soil that are consistent with those resulting from space-weathering processes occurring on airless bodies e.g., [2,5]. We hypothesize the $\mathrm{npFe}$ formed via the reduction of $\mathrm{Fe}^{2+}$ in the glassy matrix by $\mathrm{H}$ implanted by solar wind irradiation, via the following reaction, first proposed by [1]:

$$
\text { (1) } \mathrm{FeO}_{(\mathrm{s})}+2 \mathrm{H} \rightarrow \mathrm{Fe}_{(\mathrm{s})}+\mathrm{H}_{2} \mathrm{O}_{(\mathrm{s} / \mathrm{l})}
$$

The similarity of the features in the experimental samples to naturally space-weathered soils suggests this novel technique is appropriate for simulating airless body surface processes inside the TEM.

\section{References:}

[1] B Hapke, Journal of Geophysical Research- Planets 106 (2001), p. 10,039.

[2] L. P. Keller and D. S. McKay, Geochimica et Cosmochimica Acta 61 (1997) p. 2331.

[3] Sasaki, S. et al, Nature 410 (2001) p. 555.

[4] M. Loeffler et al, Journal of Geophysical ResearchPlanets (2009) 114:E03003.

[5] T. Noguchi et al, Meteoritics and Planetary Science 49 (2014) p. 188.

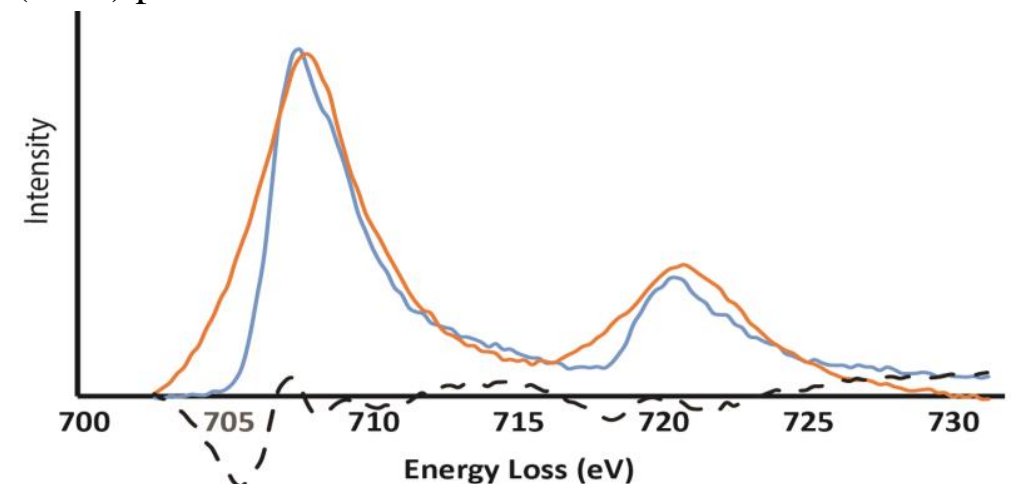

Figure 2: EELS spectra showing the measured nanoparticle in orange, and the simulated fit in blue, from a linear, least-squares fitting routine. Residuals shown in black dashed line. This nanoparticle is $100 \% \mathrm{Fe}^{0}$.
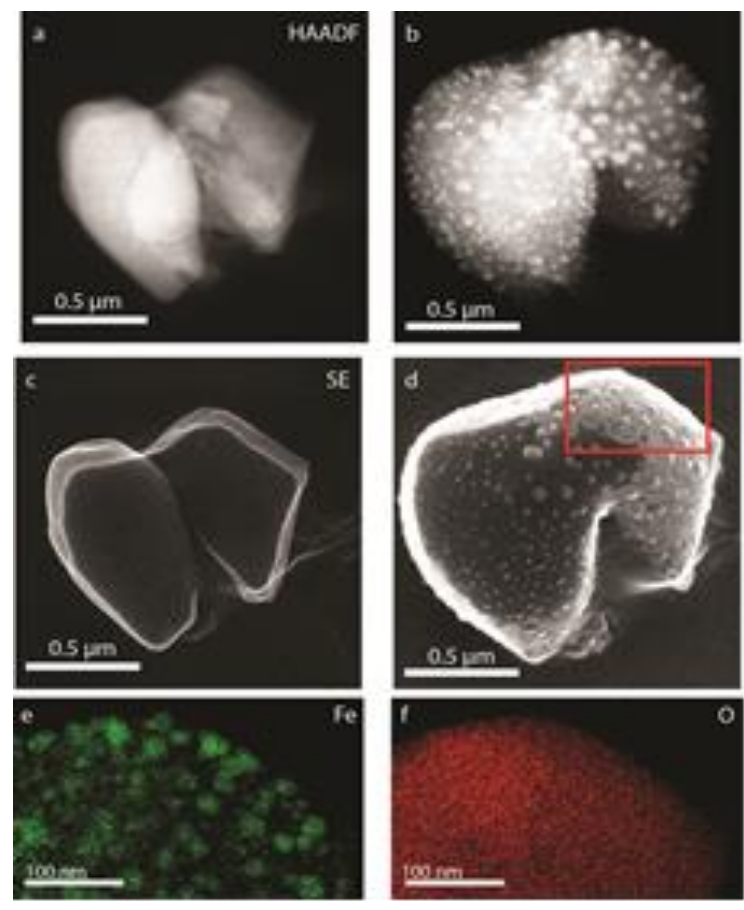

Figure 1: Images of the grain before heating and after heating, respectively, in, a,b) HAADF, c,d) SE. e,f) Fe and O EDS maps from the region outlined in the red box in d). 